\title{
Sobre el contacto y los contactos
}

\author{
Algunas reflexiones a partir del análisis de los sistemas \\ pronominales átonos de zonas de contacto lingüístico
}

\section{Introducción}

Los estudios sobre cambio lingüístico inducido por contacto publicados en las últimas décadas han sido de especial relevancia para configurar un marco teórico de la lingüística de contacto que se adapte mejor a los datos reales de las ecologías lingüísticas complejas. En el caso de los estudios sobre español, se ha superado la tendencia a abordar los fenómenos de contacto a partir de recolecciones de datos idiosincrásicas y sin rigor metodológico, con descripciones superficiales y en muchos casos parciales, con una concepción de las variaciones y los cambios inducidos por contacto como desvíos de la norma estándar o, sencillamente, como errores debidos a un aprendizaje del español incompleto. Uno de los riesgos que esto conlleva es que la estigmatización del colectivo indígena por sus hábitos lingüísticos se produce desde su ingreso en la escuela, etiquetado como "deficitario cognitivamente" o como "hablantes que no saben hablar castellano", y culpabilizados por su bajo rendimiento educativo. Y esta evaluación negativa retroalimenta la consideración de los cambios lingüísticos inducidos por contacto como simples errores lingüísticos. No olvidemos, no obstante, que bajo la etiqueta de "cambios inducidos por contacto" caben casos de importación de material y patrones fónicos, morfosintácticos, léxicos o semánticos, así como casos en los que no existe tal importación, sino que el contacto con otra lengua tiene como efecto evoluciones diferentes de variaciones y procesos de gramaticalización ya iniciados previamente.

Como es bien sabido, las situaciones de contacto de lenguas son complejas y dinámicas, y son resultado de una multitud de factores sociales y lingüísticos cuya influencia es difícil de separar, como ya apuntó en su momento Thomason (2001), lo que hace más complicado el estudio de estas ecologías lingüísticas.

Agradecimientos: Investigación realizada en el proyecto de investigación de excelencia "COREC. Oral reference corpus of Spanish in contact. Phase I: minority languages". Reference / AEI / PID2019 / 105865GB-I00. Agradezco a Sara Gómez Seibane sus valiosos comentarios que, sin duda, han contribuido a mejorar estas páginas.

Azucena Palacios, Universidad Autónoma de Madrid, azucena.palacios@uam.es, https://orcid.org/0000-0002-9122-4362

¿ Open Access. (c) 2021 Azucena Palacios, published by De Gruyter. (cc) BY-NC-ND This work is licensed under the Creative Commons Attribution-NonCommercial-NoDerivatives 4.0 International License. 
En este sentido, recordemos que una máxima de la investigación es la comparabilidad de situaciones para poder extraer generalizaciones acerca del contacto lingüístico, lo que no es una tarea fácil, sobre todo, si no se delimitan bien los parámetros de comparación de las situaciones de contacto. En efecto, no todas las situaciones de contacto lingüístico son equiparables. Imaginemos una comunidad donde haya hablantes monolingües de lengua $\mathrm{A}$, bilingües de distinto grado y monolingües de lengua B. Pero esta ecología no tiene por qué ser idéntica a la que se pueda dar en otra zona geográfica, aunque las lenguas implicadas sigan siendo A y B, ya que en esta última ecología puede que solo existan hablantes monolingües de lengua $\mathrm{B}$ y bilingües con predominio de lengua B. Y puede que la historia de contacto de ambas comunidades tampoco sea la misma. La comparabilidad de ambas ecologías resulta compleja, por tanto, dado que hay factores sociales diferentes que pueden explicar que las variaciones y los cambios inducidos por contacto en una y otra comunidad no sean exactamente los mismos.

Por otra parte, el foco de la investigación se centra en exceso en "identify the constraints involved as well as the paths such contact can take" (Siemund y Kintana 2008:3), obviandootrosaspectos esenciales. Enmuchoscasos, siguenfuncionando ciertas ideas preconcebidas sobre el contacto lingüístico que los resultados de las investigaciones basadas en datos reales de habla pueden ayudar a matizar. Esto es lo que motiva estas páginas.

En la sección 2 presento los cambios lingüísticos que experimentan los sistemas pronominales átonos de tercera persona de las variedades de español en contacto con distintas lenguas amerindias para mostrar que estas variaciones son sistemáticas y se consolidan en tendencias que cristalizan en cambios lingüísticos inducidos por contacto que se apartan de los patrones que rigen los sistemas pronominales de variedades de español de áreas sin contacto. Describo dos vías de cambio distinto al respecto: en la sección 2.1 muestro que los cambios que se documentan en los sistemas pronominales átonos de las variedades de español en contacto con maya yucateco, otomí, tepehuano, tsotsil, malecu, tzutujil o quechua tienden a neutralizar los rasgos de género y número de las formas pronominales en una única forma lo para objeto directo indistintamente de si el referente del objeto directo es masculino o femenino, singular o plural. En 2.2 ilustro cómo los sistemas pronominales átonos de las variedades de español en contacto con kichwa ${ }^{1}$ (Ecuador) y guaraní (Paraguay y Nordeste argentino) se caracterizan por un marcado leísmo donde la selección pronominal es insensible a los rasgos de caso, género e incluso número. En la sección 3 propongo una reflexión sobre los factores lingüísticos y extralin-

1 Así se denomina a la variedad de la lengua quechua ecuatoriana. Esta grafía corresponde al alfabeto oficial de la lengua. 
güísticos que configuran y explican esos cambios, y las vías de gramaticalización que han podido seguir. Concluiré esta investigación con algunas implicaciones teóricas y metodológicas que pueden extraerse de estas reflexiones.

\section{Los sistemas pronominales de las variedades de contacto: cambios indirectos inducidos por contacto}

\subsection{Sistemas con neutralización de género/número y mantenimiento de caso}

Como ya se ha mostrado sobradamente (Avelino 2017; Calvo 1996-1997; García Tesoro 2010, García Tesoro 2018; García Tesoro y Fernández-Mallat 2015; Godenzzi 1986; Hernández y Palacios 2015; Klee 1990; Klee y Caravedo 2005; Martínez 2000, Martínez 2013, Martínez 2015a; Palacios 2005, Palacios 2006, Palacios 2011; Sánchez Avendaño 2015; Sánchez Paraíso 2017; Torres Sánchez 2015, Torres Sánchez 2018; entre otros), los sistemas pronominales átonos de las variedades de español en contacto con lenguas originarias de Hispanoamérica muestran variaciones y cambios lingüísticos en los que se produce una aparente simplificación de rasgos morfológicos en las formas pronominales que afectan al género, al número y, en menor medida, al caso. Se ha constatado, igualmente, que estas variaciones no son caóticas o aleatorias y que se consolidan en tendencias que cristalizan en cambios lingüísticos inducidos por contacto que se apartan de los patrones que rigen los sistemas pronominales átonos de variedades de español de áreas sin contacto.

Estos sistemas pronominales locales coexisten con el sistema pronominal etimológico, que es el normativo, y que se exige en el ámbito escolar, aparece en los medios de comunicación - salvo en el caso del español andino ecuatoriano o paraguayo, donde incluso en estos medios se puede documentar el sistema pronominal local si se trata del registro oral coloquial o informal - y en los ámbitos donde se requiere el manejo de un español prestigioso. Esto supone, en mayor o menor medida, una presión normativa sobre los sistemas pronominales locales, que se traduce en cierta variabilidad de la frecuencia de uso de las formas pronominales que oscilan entre ambos patrones: el etimológico y el local. Como veremos más adelante, los hablantes que muestran frecuencias de uso más altas de estos sistemas locales son, en general, los bilingües con lengua originaria dominante, los que tienen menor nivel de escolarización y los que por sus circunstancias so- 
ciales, por ejemplo, por cuestiones profesionales, están menos sometidos a la presión de la norma lingüística que establece que el sistema pronominal átono de tercera persona "correcto" es el etimológico. Estamos hablando, por tanto, de tendencias de uso. No obstante, aunque estos son los lineamientos generales, las circunstancias e historias sociolingüísticas de las distintas comunidades pueden variar las frecuencias de uso de las formas locales y etimológicas.

En esta sección expongo que los cambios que se documentan en los sistemas pronominales átonos de las variedades de español en contacto con maya yucateco, otomí, tepehuano, tsotsil, malecu, tzutujil o quechua son cambios indirectos inducidos por contacto que forman parte de un proceso de gramaticalización en curso; cambios sistemáticos en los que opera el mecanismo de la convergencia lingüística (Palacios 2005). Estas lenguas originarias americanas no pertenecen a las mismas familias lingüísticas, a pesar de lo cual las soluciones innovadoras que muestran sus hablantes son similares y suponen un sólido argumento que avala la existencia del mencionado proceso de gramaticalización, esto es, un proceso general y sistemático de cambio donde la congruencia estructural de las lenguas en contacto condiciona las soluciones emergentes.

Para mostrar que estas afirmaciones son argumentos sólidos a favor de la propuesta, he tomado, y en algún caso adaptado, los datos que ofrecen algunas investigaciones sobre las variedades de español en contacto realizadas a partir de datos de habla real. Como se aprecia en (1-7), las formas pronominales átonas de tercera persona de estas variedades propenden a neutralizar los rasgos de género y número de las formas pronominales, tendiendo hacia una única forma lo para objeto directo:

(1) a. Porque esas iglesias que se han construido, lo han. . . lo han construido los norteamericanos ${ }^{2}$.

b. Dos banquillos agarro así. . .lo pongo así. Me paro a moler.

(2) a. La muchacha tantito que lo regañan ahorita ya no se halla, ya se va. ${ }^{3}$

b. Entonce crecía esos animalitos, entonce pus lo vendía.

(3) a. La chuparrosa lo mandaron a derechamente (<directamente) allá donde está la lluvia ${ }^{4}$.

b. Ahora tienes que lavarlo estas dos muñecas.

2 Datos del español en contacto con maya yucateco tomados de Hernández y Palacios (2015: 36).

3 Datos del español en contacto con otomí tomados de Avelino (2021: 78).

4 Ejemplos de español en contacto con tepehuano tomados de Torres Sánchez (2018: 228). 
(4) a. Y al final le pongo su hoja de laurel y arrayán y lo tapo la olla ya que se cuece con todo y su hoja. ${ }^{5}$

b. Pero le podemos pedir favor alguna/algunas preguntas/lo pones en un papel.

(5) a. Pobrecita iguanita tal vez anda abajo ahí y lo agarran para comer. ${ }^{6}$

b. Agarraron unas muchachas/tal vez de/digo yo que tal vez quince años/y el papá decía que no lo/que no lo agarre que no lo no tiene por qué violarlo.

(6) a. En el monte así, consigue esa hierba y lo trae, lo trae comprada o regalada, viene a que lo lave bien. ${ }^{7}$

b. Las tradiciones lo practican la gente ladina y nuestra raza más que todo ahí en la comunidad.

(7) a. En Chinchero más lo preparan la chicha, pero acá así cuando hay cualquier cosa no más lo preparan. ${ }^{8}$

b. Es un, son hojitas verdes que lo picas así picaditas, lo cocinan, lo hacen su [.. .], con ajito y cebollita, con comino, lo mezclan, y sale, como un. . .

Lo interesante de estas soluciones es que no se trata de casos esporádicos o anecdóticos que aparecen de vez en cuando en la variedad oral de los hablantes, sino que forman parte de una reorganización del sistema que afecta en mayor o menor medida a los usos pronominales. Veamos algunos datos cuantitativos que apoyan esta argumentación.

Hernández y Palacios (2015) llevan a cabo un exhaustivo estudio sobre los sistemas pronominales átonos de la zona maya yucateca. Tras un análisis multivariado, las autoras determinan las relaciones de dependencia entre variables y la significancia de las mismas, y aportan datos de frecuencia relativa de uso de las formas pronominales de objeto directo de hablantes monolingües de español y bilingües de español y maya yucateco. Para los fines de este trabajo, mostramos únicamente la tabla donde las autoras contabilizan el uso de lo con referentes femeninos de objeto directo: ${ }^{9}$

5 Datos de español en contacto con tsotsil tomados de López Gutiérrez (2018: 36 y 38).

6 Datos de español en contacto con malecu tomados de Sánchez Avendaño (2015: 89).

7 Ejemplos de español en contacto con tzutujil tomados de García Tesoro (2010: 139).

8 Ejemplos de español en contacto con quechua tomados de García Tesoro y Fernández-Mallat (2015: 133).

9 En esta variedad, los objetos directos con referentes masculinos se pronominalizan mediante lo/s. En el conjunto de la muestra, los referentes femeninos se pronominalizan con $l o / s$ en el $53 \%$ de los casos; la/s suponen el 35,6 \% de las apariciones totales y le/s el 5,3\%. 
Tabla 1: Usos de la forma lo con referente femenino y factor bilingüismo.

\begin{tabular}{ll|l}
\hline & Lo con referentes femeninos \\
\hline Monolingües & $19,2 \%$ & \\
\hline Bilingües dominio español & $31,3 \%$ & \\
\hline Bilingües equilibrado & $38,9 \%$ & \\
\hline Bilingües dominio maya & $85,4 \%$ & \\
\hline
\end{tabular}

Hernández y Palacios constatan así que existe una tendencia de uso de la forma de acusativo lo sin especificación de género, indistintamente de si el referente es masculino o femenino, y que esta tendencia está asociada con el dominio de la lengua maya; esto es, los hablantes que tienen la lengua maya como dominante son los que muestran mayores porcentajes de uso de lo con referentes femenino, el 85,4\%; por el contrario, los hablantes que no saben maya tienen el 19,2\% de los casos de usos de lo con referentes femeninos, una cifra nada desdeñable, no obstante. Nótese que los hablantes con dominio de español muestran un 31,3\% de usos de esta forma también, que se incrementa entre los bilingües equilibrados hasta el 38,9\%. Inversamente, los hablantes monolingües de español tienen el mayor porcentaje de usos etimológicos, 81,8 \% de la para referentes femeninos, frente a los bilingües con dominio de la lengua maya, con la menor frecuencia $(14,6 \%)$.

Esta gradación porcentual es altamente significativa y permite a las autoras consolidar su hipótesis de partida: la reorganización del sistema pronominal átono hacia formas de concordancia de objeto: lo para objeto directo y le para objeto indirecto. ${ }^{10}$ Para poder vislumbrar el alcance y dirección del cambio, analizan la frecuencia relativa de la forma lo también con referentes plurales (Tabla 2), constatando que la asociación de las variables "dominio de la lengua maya" y "uso de la forma lo" es también significativa y guarda la misma relación gradual que vimos en la Tabla 1, si bien los porcentajes de uso son ligeramente inferiores.

10 Hernández (2017) documenta la siguiente pronominalización de los objetos indirectos en su muestra: una única forma la $(0,3 \%)$, la forma lo $(4,2 \%)$ y le/s en el resto. No registró casos de pronominalización del objeto indirecto con las ni con los. La autora constata, además, una tendencia a usar la forma le para objetos indirectos con referentes plurales en esta misma variedad: $61,9 \%$ en bilingües con dominio de maya, $36 \%$ en simétricos, 31,6 \% en bilingües con dominio de español y $31,6 \%$ en monolingües de español. 
Tabla 2: Usos de la forma lo con referente plural y factor bilingüismo.

\begin{tabular}{ll|l}
\hline & Lo con referentes plurales \\
\hline Monolingües & $13,2 \%$ & \\
\hline Bilingües dominio español & $15,9 \%$ & \\
\hline Bilingües equilibrado & $31,3 \%$ & \\
\hline Bilingües dominio maya & $64,9 \%$ & \\
\hline
\end{tabular}

En efecto, los hablantes bilingües con dominio de la lengua maya tienen frecuencias de uso muy altas de lo con referentes plurales (64,9\%). Existe, además, una gradación en el uso de esta forma relacionada con el mayor dominio de la lengua originaria. Por otra parte, las autoras constatan en su estudio que el grado de asociación de las variables "género del referente y factor bilingüismo" (coeficiente de contingencia 0,491; V. de Cramer 0,563; N=124) es ligeramente mayor que el de las variables "número del referente y factor bilingüismo" (coeficiente de contingencia 0,429; V. de Cramer 0,475; N=135), lo que indica que la neutralización de género está en una etapa de evolución más avanzada que la de número. Se puede afirmar, por ello, que en esta variedad de español coexisten dos sistemas que no están aislados, sino que forman parte de un continuo gradual y que la variable "dominio de la lengua maya/dominio del español" regula los porcentajes de uso de ese continuo, mostrando el predominio de uno $u$ otro sistema. Los Cuadros 1 y 2 representan los sistemas pronominales ideales, ${ }^{11}$ con patrones de género, número y caso en el sistema etimológico y solo de caso en el sistema local.

Cuadro 1: Sistema etimológico.

\begin{tabular}{lllll}
\hline & \multicolumn{2}{c}{ Masculino } & \multicolumn{2}{c}{ Femenino } \\
\hline & Sg. & Plural & Sg. & Plural \\
\hline OD & lo & los & la & las \\
\hline & & Singular & \multicolumn{2}{c}{ Plural } \\
\hline Ol & & le & les \\
\hline
\end{tabular}

11 Es preciso recordar que los patrones ideales no existen en ninguna variedad de español y que la variación está presente desde la Edad Media en nuestra lengua. 
Cuadro 2: Sistema local.

\begin{tabular}{|c|c|c|c|c|}
\hline & \multicolumn{2}{|c|}{ Masculino } & \multicolumn{2}{|c|}{ Femenino } \\
\hline & Sg. & Plural & Sg. & Plural \\
\hline \multirow[t]{2}{*}{ OD } & & 10 & & \\
\hline & & Singular & & Plural \\
\hline OI & & le & & \\
\hline
\end{tabular}

Según hemos visto, en esta variedad de español en contacto surge un nuevo sistema pronominal donde lo es la forma emergente sin especificación de género o número para el objeto directo y le para el indirecto. Este sistema coexiste con el sistema normativo etimológico y el predominio de uso de uno u otro se favorece en función de la lengua dominante.

Los datos de otras variedades de contacto que mostramos a continuación, simplificados por razones de espacio, ${ }^{12}$ ofrecen un panorama de cambio similar que constatan su sistematicidad. Así, los porcentajes de uso de las formas pronominales de la variedad de español en contacto con otomí en la comunidad San Andrés Cuexcontitlán (México), donde el otomí está en una situación de desplazamiento, se presentan en la Tabla 3 (Avelino 2017).

Tabla 3: Usos de la forma lo/s con referente femenino y factor bilingüismo.

\begin{tabular}{ll|l}
\hline & Lo/s con referente femenino \\
\hline Monolingüe español (sin conocimiento otomí) & $5,74 \%$ & \\
\hline Monolingüe español conocimiento pasivo otomí & $16,60 \%$ & \\
\hline Bilingües con dominio de español & $60 \%$ & \\
\hline Bilingües equilibrado & $77,27 \%$ & \\
\hline Bilingües con dominio de otomí & $95,45 \%$ & \\
\hline
\end{tabular}

Como indican las frecuencias, el patrón gradual que mostramos en la variedad de español en contacto con maya yucateco se confirma: a mayor dominio de la lengua

12 Los datos que presentamos en las tablas siguientes están elaborados a partir de análisis cuantitativos y cualitativos, según los cuales, las variables bilingüismo y forma pronominal lo/s con referente femenino están correlacionadas. Mostramos únicamente los datos relativos a la neutralización del rasgo género, esto es, usos de lo y los para referentes femeninos, tanto singulares como plurales. Evidentemente, los objetos con referentes masculinos se pronominalizan con lo/s. Como ya he señalado, la neutralización del rasgo de número en las formas pronominales se constata como un cambio menos avanzado que el del género. 
originaria, mayor uso de formas sin especificación de género. Es ciertamente relevante que el porcentaje de usos de lo/s con referentes femeninos en hablantes monolingües de español sin conocimiento del otomí sea sensiblemente inferior (5,74 \%) al de los monolingües con algún conocimiento pasivo de otomí (16,60\%), si bien en ambos casos se trata de hablantes monolingües de español. Este porcentaje aumenta considerablemente en los bilingües con dominio de español (60\%), que sube hasta el 77,27 \% cuando los hablantes bilingües son simétricos y hasta el 95,45 \% en el caso de los hablantes bilingües con dominio de otomí. Avelino (2017, 2021) concluye que en esta comunidad coexisten dos sistemas: a) el sistema etimológico, predominante en los monolingües de español y en los hablantes con conocimiento pasivo de otomí y bilingües con dominio de español; b) el sistema local, predominante en los hablantes bilingües con dominio de otomí.

En la Tabla 4 mostramos datos de la variedad de español en contacto con tzutujil en Guatemala (García Tesoro 2010, 2018). El trabajo de campo se realizó en Chicacao, una zona que comprende un núcleo urbano donde convive población no indígena e indígena, y núcleos rurales donde predomina la población indígena. Los colaboradores tienen diferentes niveles de instrucción a excepción del grupo de bilingües con dominio de tzutujil, que no han tenido acceso a la escolarización.

Tabla 4: Usos de la forma $l o / s$ con referente femenino y factor bilingüismo.

\begin{tabular}{ll|l}
\hline & Lo/s referente femenino \\
\hline Monolingües español sin contacto con tzutujil & $4,2 \%$ & \\
\hline Monolingües español con contacto con tzutujil & $33,3 \%$ & \\
\hline Bilingües simétricos & $84 \%$ & \\
\hline Bilingües con dominio de tzutujil & $100 \%$ & \\
\hline
\end{tabular}

Es interesante constatar cómo el sistema local predomina entre hablantes bilingües simétricos (84\%) y con dominio de tzutujil (100\%), pero resulta sumamente relevante que los hablantes monolingües de español que viven en ambientes bilingües usan la forma local lo/s con referentes femeninos en el 33,3\% de los casos; evidentemente, la mayoría de sus usos corresponde al patrón etimológico, pero ese $33,3 \%$ es un indicio de que en su entorno hay una variedad local estable donde ambos sistemas pronominales conviven (compárese con el 13,2 \% de usos locales en los monolingües de español de la zona maya yucateca).

El caso de la variedad de español en contacto con tepehuano del sureste, que mostramos en la Tabla 5 (Torres Sánchez 2018), si bien ofrece datos donde se aprecia un patrón similar de uso de las formas pronominales, es ligeramente 
diferente debido a que se trata de una comunidad con bilingüismo reciente y no existen hablantes monolingües de español en la zona de contacto.

Tabla 5: Usos de la forma lo/s con referente femenino y factor bilingüismo.

\begin{tabular}{ll|l}
\hline & \multicolumn{2}{l}{ Lo/s referente femenino } \\
\hline Monoling. español sin contacto tepehuano & $8,08 \%$ & \\
\hline Bilingües + conocimiento español & $62,7 \%$ & \\
\hline Bilingües +/ - conocimiento español & $70,3 \%$ & \\
\hline Bilingües con - conocimiento español & $83,3 \%$ & \\
\hline
\end{tabular}

En la variedad mexicana de San Cristóbal de las Casas en contacto con tsotsil, López Gutiérrez (2018) ofrece solo datos de monolingües y bilingües de la misma zona sin especificar el grado de conocimiento de cada lengua, como se muestra en la Tabla 6.

Tabla 6: Usos de la forma $l o / s$ con referente femenino y factor bilingüismo.

\begin{tabular}{|c|c|}
\hline & Lo/s referente femenino \\
\hline Monolingües español & $56,6 \%$ \\
\hline Bilingües & $65,24 \% \nabla$ \\
\hline
\end{tabular}

La muestra de la Tabla 6 refleja la conformación sociolingüística de la comunidad, caracterizada por la migración de población indígena a la ciudad. Los monolingües de español son personas que han nacido en San Cristóbal, los bilingües pueden ser oriundos de la ciudad o haber migrado desde comunidades tsotsiles cercanas. En ambos grupos hay colaboradores con niveles de instrucción semejantes. Es sumamente interesante constatar que, en esta variedad, los hablantes monolingües de español tienen un porcentaje altísimo de uso de $l o / s$ con referentes femeninos (56,6 \%), si bien los hablantes bilingües llegan hasta el 65,24\%. Esto parece apuntar a una variedad de español bastante estable en la que el sistema pronominal local parece predominar sobre el etimológico, al menos en el registro oral coloquial.

Los datos que ofrece Sánchez Avendaño (2015) de español en contacto con malecu son excepcionales, dado que diferencia los usos de las formas pronominales en función de la edad de los colaboradores en los tres palenques o comunidades malecu. A partir de estos datos, se ha elaborado la Tabla 7, donde se muestran los resultados de los entrevistados de más de 50 años. 
Tabla 7: Usos de la forma lo/s con referente femenino y factor bilingüismo (+ 50 años).

\begin{tabular}{lcll}
\hline & Margarita & El Sol & Tonjibe \\
\hline Biling. equilibrado & $36 \%$ & $60 \%$ & $\cdots$ \\
\hline Biling. malecudominante & $100 \%$ & $\checkmark---$ & $100 \%$ \\
\hline
\end{tabular}

La Tabla 7 sigue el patrón gradual de usos de lo/s con referentes femeninos en función del dominio de la lengua: los hablantes bilingües con malecu dominante usan mayoritariamente el sistema pronominal local, que baja notablemente en los bilingües equilibrados, 36 \% en Margarita y 60 \% en El Sol. Sánchez Avendaño ofrece también datos de colaboradores bilingües dominantes de español con algún grado de conocimiento de malecu menores de 30 años en esos mismos palenques (Tabla 8).

Tabla 8: Usos de la forma lo/s con referente femenino (- 30 años).

\begin{tabular}{lrrr}
\hline & Margarita & El Sol & Tonjibe \\
\hline Esp. dominante & $24,3 \%$ & $60 \%$ & $59,6 \%$ \\
\hline
\end{tabular}

Los datos divididos por edades permiten observar el estado de desplazamiento de la lengua malecu en los tres palenques, pero también cómo estos hablantes jóvenes siguen mostrando un porcentaje significativo de usos de $l o / s$ con referente femenino. Margarita es el que muestra porcentajes de uso de $l o / s$ con referente femenino más bajos, lo que puede deberse a que es el palenque con mayor desplazamiento de malecu - la primera escuela se instaló en este palenque a mediados del siglo pasado -. No obstante, las frecuencias de uso confirman, como afirma Sánchez Avendaño (2015: 94) que "no se trata de variaciones propias de la interlengua de aprendices tardíos del español, sino de un proceso de cambio a favor de lo con referente femenino".

\subsection{Sistemas leístas con neutralización de género/ número y caso}

La bibliografía ha mostrado que en las variedades de contacto con guaraní (Paraguay y Nordeste argentino) y en el español andino ecuatoriano (de bilingüismo histórico español/kichwa) los sistemas pronominales átonos locales se caracterizan por un marcado leísmo donde la selección pronominal es insensible a los rasgos de caso, género e incluso número (De Granda 1982; Guillán 2012; Haboud 
y de la Vega 2008; Jarrín 2019; Martínez 2000, Martínez 2013; Palacios 1998a, Palacios 2005, Palacios 2015; Symeonidis 2013; Yausaz 2005). En el Cuadro 3 se muestra el patrón pronominal leísta.

Cuadro 3: Sistema leísta.

\begin{tabular}{|c|c|c|}
\hline & Masculino & Femenino \\
\hline$\overline{O D}$ & le/les & \\
\hline $\mathrm{OI}$ & le/les & \\
\hline
\end{tabular}

Los datos de Formosa (8), de Paraguay (9) y de la sierra ecuatoriana (10) que presentamos a continuación siguen el sistema leísta.

(8) a. Y porque le quisieron alistar a mis hermanos. ${ }^{13}$

b. Mientras vo armaste la torta poné agua que se hierva, esa agua hirviendo le derramás arriba de esa torta que hiciste ¿no?

c. Por ejemplo le ponían la mandioca en el sol. Se seca y depué le machaca.

d. Lo que hicieron a la chica de Cubas, que le secuestraron y le tenían en pleno centro de Asunción.

(9) a. En loh Etadoh Unidoh por ejemplo loh norteamericanoh le tienen como animaleh [a los indios]. ${ }^{14}$

b. Siempre vah y le saludah a tu padrino.

c. Siempre le ehtamoh hasiendo [la sopa paraguaya].

d. La polisía le desalojó el lugar.

(10) a. Y a mi esposa solo le veía sábado y el domingo [risas] ${ }^{15}$.

b. O sea que ahí tendríamos que acordarnos para poder visitarles a ellos.

c. ¿La fritada? Le matan al chancho y no sé no sé con la carnecita más pura, como pechuga de pollo...

d. Si hay un atasco, le utiliza la señora el claxon y, si no cruzas breve la calle, te pitan.

13 Datos tomados de Guillán (2012).

14 Tomados de Palacios (2002).

15 Datos tomados del Corpus Oral de Referencia del Español en Contacto (COREC). http://espanolcontacto.fe.uam.es/wordpress/muestras-corec/. 
En Formosa el leísmo no es una variante prestigiosa en la zona. Al respecto, Guillán (2012) analiza tres grupos de hablantes con perfiles sociolingüísticos diferentes y concluye que en todos ellos coexisten el sistema etimológico y el leísta, pero en proporciones diferentes según su perfil sociolingüístico, como se aprecia en la Tabla 9.

Tabla 9: Sistemas pronominales en Formosa.

\begin{tabular}{|c|c|c|}
\hline & Sistema etimológico & Sistema leísta \\
\hline Monolingües & $90,3 \%$ & $9,7 \%$ \\
\hline Monoling. ambiente bilingüe & $61,9 \%$ & $38,1 \%$ \\
\hline Bilingües & $5,3 \%$ & $94,7 \%$ \\
\hline
\end{tabular}

Para la variedad de español correntino en el Nordeste argentino, Yausaz (2005) hace un análisis muy interesante de producciones orales y escritas en niños de Goya y Corrientes capital. Muestra cómo en Goya el leísmo aparece en el $100 \%$ de los casos en las producciones orales, pero en el $70 \%$ de las escritas. En Corrientes capital, el leísmo llega al $87 \%$ de los usos pronominales totales en las producciones orales mientras que en las escritas solo alcanza el $58 \%$. El autor considera que esta asimetría se debe a la evaluación negativa que hacen los docentes correntinos del leísmo en la escuela y al poco prestigio que tiene el fenómeno en la zona, de manera similar a lo que veíamos en Formosa. Considera Yausaz (2005: 6) al respecto que "para los niños de la escuela de Corrientes, la identificación de situaciones comunicativas en las cuales es adecuado utilizar la variedad estándar [el sistema etimológico] no resulta una tarea sencilla”.

En el caso de Paraguay, también el sistema leísta es el predominante en la variedad oral como ha constatado la bibliografía (Palacios 1998a, Palacios 2000b, Palacios 2005, Palacios 2008; Symeonidis 2013; entre otros). La tendencia al uso de un único pronombre le para objeto directo e indirecto sin distinción de género e incluso de número no supone un cambio estigmatizado y se generaliza entre la población urbana de nivel medio y medio-alto, al menos en el registro oral. Symeonidis (2013: 66), a partir de un estudio con 86 colaboradores, afirma que "se muestra un empleo general del leísmo en el castellano del Paraguay, un leísmo que sorprendentemente tiene como casi única forma el pronombre personal le tanto para referentes en el singular como en el plural. En los pocos ejemplos que hemos visto en los que no se usa la forma le sino una forma etimológica, se observa que es la clase alta la que tiende de vez en cuando a usar dichas formas". 
En el caso del español andino ecuatoriano, el sistema leísta es mayoritario en el registro oral, sobre todo en Quito, y se ha convertido en un fenómeno prestigioso tanto en monolingües como en bilingües (Haboud y de la Vega 2008, Palacios 2005, Palacios 2006, Palacios 2015), mientras que el etimológico se circunscribe al registro escrito, por ejemplo, la prensa, sobre todo, en hablantes con nivel medio-alto de instrucción. ${ }^{16}$ Para los fines de este estudio, he realizado un muestreo representativo con datos del corpus COREC. He seleccionado los usos pronominales de objeto directo de 5 colaboradores monolingües y 5 bilingües de la sierra ecuatoriana en dos puntos: Quito y Otavalo. El resultado constata el leísmo mayoritario de los hablantes monolingües y bilingües - tanto con referentes masculinos como femeninos -, si bien el porcentaje de leísmos en los bilingües es sensiblemente inferior, como se muestra en la Tabla 10.

Tabla 10: Sistema leísta en la sierra ecuatoriana.

\begin{tabular}{llll}
\hline & Le $/ \mathbf{s}$ & Lo/s & La/s \\
\hline Monolingües & $89,8 \%$ & $10,2 \%$ & $0 \%$ \\
\hline Bilingües & $84,6 \%$ & $14,8 \%$ & $0,6 \%$ \\
\hline
\end{tabular}

Como ya se ha constado en la bibliografía (Palacios 2015), los dos grupos de hablantes realizan omisiones de objeto directo (Tabla 11).

Tabla 11: Formas pronominales y omisión.

\begin{tabular}{lrr}
\hline & Formas pronominales & Omisión \\
\hline Monolingües & $69,5 \%$ & $30,5 \%$ \\
\hline Bilingües & $57,9 \%$ & $42,1 \%$ \\
\hline
\end{tabular}

El $100 \%$ de las omisiones de objeto directo efectuadas por los monolingües y el $96,7 \%$ de los bilingües tienen referentes inanimados. Esto significa que la omisión se favorece con el rasgo animacidad. Este mismo rasgo es el que articula la selección de las formas le/s, como se muestra en la Tabla 12.

16 Esto no indica que no existan hablantes con sistema etimológico o con variación entre el etimológico y el leísta en Quito, sobre todo en población con alto nivel de instrucción; sin embargo, en los diferentes trabajos de campo que he realizado, al menos, no lo he podido constatar. 
Tabla 12: Rasgo animacidad y formas le/s.

\begin{tabular}{lrr}
\hline & + Animado & - Animado \\
\hline Monolingües & $76,1 \%$ & $23,9 \%$ \\
\hline Bilingües & $65 \%$ & $35 \%$ \\
\hline
\end{tabular}

En la Tabla 13 se muestran los porcentajes de uso de las formas lo/s en función del rasgo de animacidad.

Tabla 13: Rasgo animacidad y formas lo/s.

\begin{tabular}{lrr}
\hline & + Animado & - Animado \\
\hline Monolingües & $30 \%$ & $70 \%$ \\
\hline Bilingües & $4 \%$ & $96 \%$ \\
\hline
\end{tabular}

Comparando las frecuencias de uso de las formas le/s y lo/s en función del rasgo de animacidad se constata que los referentes inanimados favorecen la selección de $l o / s$, sobre todo en los hablantes bilingües (96\%). Vale la pena comprobar si los usos de lo/s corresponden a un patrón etimológico en ambos grupos, esto es, si el género masculino del referente es el que favorece la selección de estas formas minoritarias. En la Tabla 14 se muestran los resultados.

Tabla 14: Formas lo/s y género del referente.

\begin{tabular}{lrr}
\hline & Masculino & Femenino \\
\hline Monolingües & $100 \%$ & $0 \%$ \\
\hline Bilingües & $69,7 \%$ & $30,3 \%$ \\
\hline
\end{tabular}

Los datos son contundentes: los hablantes monolingües seleccionan $l o / s$ si el referente es únicamente masculino, lo que indica que siguen un patrón etimológico cuando seleccionan esa forma. Sin embargo, los bilingües seleccionan la forma $l o / s$ con referentes masculinos $(69,7 \%)$ y femeninos (30,3\%). Volveremos sobre ello más adelante. En cualquier caso, no olvidemos que esas formas $l o / s$ son minoritarias (10,2 \% en monolingües, $14,8 \%$ en bilingües) y que el patrón mayoritario en ambos colectivos es el leísta, lo que coincide con los datos del estudio de Jarrín (2019: 37) en el norte de Quito, que concluye que el leísmo es el único sistema documentado en su muestra y que este sistema "se ha consolidado ya incluso en zonas cuyos habitantes crecieron en un entorno bilingüe quichua-castellano, pero que hoy por hoy son mayoritariamente monolingües en español”. 
Se podría pensar que el sistema leísta es un cambio analógico que se origina porque las formas acusativas copian directamente a las de dativo; sin embargo, hay evidencias de un sistema pronominal de transición entre el sistema etimológico y el leísta en las variedades andinas ecuatorianas (11) y paraguayas (12) que parecen contradecir esta hipótesis (Palacios 2000b, Palacios 2005, Palacios 2006, Palacios 2011):

(11) a. Que no le puedes ver [a los ecuatorianos].

b. Mi hijita se quedó en Loja, pero le traje.

c. Yo te lo voy a obsequiar [un sombrero]. Yo tengo uno que me lo mandan.

e. La feria creo que lo hacen los viernes.

(12) a. Le encontré a ellos en el monte

b. No podía dejarle a la criatura sola y mandarme mudar.

c. Un trabajo de diez personas, se van a hacerlo.

d. Esa chipa de la que te hablé, pues lo hacen de todo.

Este sistema se documentó en hablantes bilingües y monolingües de español de áreas de contacto kichwa cercanas a Quito y también en Otavalo (Palacios 2006, Palacios 2011); en el caso de Paraguay, en zonas semiurbanas cercanas a Asunción (Palacios 2000b). Son sistemas minoritarios y están en franco retroceso. En el caso ecuatoriano, la forma pronominal lo/s con referente femenino es un rasgo indexado con comunidades indígenas que tiene una categorización social negativa. No olvidemos que tanto Quito como Asunción actúan como difusores del leísmo en el registro oral. En el sistema de transición, la animacidad es un rasgo relevante, puesto que favorece la forma leísta indistintamente del género del referente, como se muestra en (11 a, b) y (12 a, b). Los referentes inanimados tienden a pronominalizarse mediante la forma $l o / s$, sin que el género del referente sea relevante en la selección pronominal, como se aprecia en (11 c-d) y (12 c-d). En el Cuadro 4 aparece representado este sistema.

Cuadro 4: Sistema de transición.

\begin{tabular}{|c|c|c|c|c|}
\hline & \multicolumn{2}{|c|}{-Animado } & \multicolumn{2}{|c|}{ +Animado } \\
\hline & Masc. & Fem. & Masc. & Fem. \\
\hline $\mathrm{OD}$ & \multicolumn{2}{|c|}{$10 / \mathrm{s}$} & \multicolumn{2}{|c|}{$l e / s$} \\
\hline $\mathrm{OI}$ & \multicolumn{4}{|c|}{$l e / s$} \\
\hline
\end{tabular}

Al respecto, volvamos a las Tablas 13 y 14, donde se analizaba ese 14,8 \% de formas lo/s documentadas en los hablantes bilingües leístas. Recordemos que 
el $96 \%$ de esos usos tenían referentes inanimados y el 30,3\% tenían referentes femeninos. Según estos resultados, esas formas minoritarias $l o / s$ parecen obedecer a un patrón similar al mostrado en el Cuadro 4.

\section{Discusión}

Los datos que hemos mostrado en $\S 2.1$ y $\$ 2.2$ permiten constatar que en las áreas de contacto lingüístico descritas conviven dos sistemas pronominales: el normativo etimológico y los locales, caracterizados por tender hacia formas pronominales que neutralizan las especificaciones de género, número y/o caso. Este hecho tiene lugar en áreas de contacto donde las lenguas originarias implicadas pueden no estar emparentadas tipológicamente (otomí, quechua, maya o malecu, por ejemplo, pertenecen a familias distintas). Lo interesante es, por tanto, que las soluciones emergentes documentadas en estas comunidades confluyen en los mismos patrones pronominales que hemos denominado sistemas locales (vid. Cuadros 2 y 3 ).

Volviendo a los casos descritos en (1-7), los efectos lingüísticos observados en los sistemas pronominales locales evidencian que se mantiene la distinción de caso y que los rasgos de género $\mathrm{y}$, en menor medida, de número, tienden a neutralizarse en favor de la forma no marcada (masculino singular). Y esta cancelación de la especificidad de los rasgos tiene lugar en función de la configuración de los grupos sociolingüísticos (mayor tendencia a la neutralización entre los que tienen la lengua originaria dominante y menor entre los monolingües de español). Así, a partir del sistema etimológico surge un nuevo sistema pronominal donde lo y le son las formas emergentes no marcadas, sin especificación de género o número, para el objeto directo y el indirecto, respectivamente.

Los estudios sobre dinámicas de adquisición de los pronombres átonos en español en niños monolingües y bilingües constatan que el paradigma pronominal se adquiere en etapas sucesivas: en primer lugar, la distinción de caso mediante las formas no marcadas lo y le; posteriormente, las distinciones de género y de número ${ }^{17}$ (Aguado Orea 2000; Domínguez 2006; Franco 2000; Klee 1989; Lafford y Collentine 1987; Pueyo 1992; Hernández Pina 1990, entre otros). Consideran que se trata de dinámicas de adquisición universales. Si esto es así, cabe preguntarse si los cambios representados en el Cuadro 2 pueden explicarse a partir de dinámicas de adquisición incompleta, lo que supondría que los

17 No hay acuerdo en la bibliografía sobre el orden de adquisición de los rasgos de género y número. 
hablantes solo habrían adquirido la primera etapa del paradigma pronominal: la distinción de caso.

Si solo nos fijamos en los grupos de hablantes bilingües con lengua amerindia dominante, podría pensarse que se trata de pautas de adquisición incompleta del paradigma pronominal. Sin embargo, esta argumentación no se sostiene al constatar que los grupos de hablantes bilingües simétricos y de monolingües de español también documentan formas pronominales locales en mayor o menor medida, por lo que parece, más bien, que se trata de soluciones que han pasado a formar parte de una variedad de español local más o menos estable. ${ }^{18}$ En efecto, los datos de las comunidades descritas en la sección 2.1 muestran que el factor bilingüismo ${ }^{19}$ es esencial para explicar la aparición de un mismo patrón gradual proporcionalmente invertido que oscila entre usos que corresponden al sistema pronominal local y usos que siguen el etimológico. Como hemos podido comprobar en las Tablas 1 a 8, este patrón gradual invertido es consistente y se repite sistemáticamente: a mayor dominio del español, menor tendencia de uso de formas locales; a mayor dominio de la lengua originaria, menor tendencia de uso de formas etimológicas. Así, los hablantes bilingües con lengua originaria dominante son los que muestran frecuencias de uso más altas del sistema local, que en el caso de hablantes de tzutujil y malecu llegan hasta el $100 \%$ de los casos. Las frecuencias de uso de las formas pronominales locales disminuyen entre los bilingües equilibrados; son menos frecuentes entre los bilingües con español dominante y la frecuencia disminuye entre los monolingües de zonas bilingües. Se trata, por tanto, de tendencias de uso en las que predomina uno u otro patrón en función de, al menos, el factor "lengua dominante".

A la vista de estos datos, podemos concluir que el cambio se origina en el grupo de los bilingües con lengua originaria dominante y se expande progresivamente hacia los otros grupos. Esa difusión intergrupal de las formas emergentes tiene lugar a partir de los objetos directos más prototípicos, al menos en la variedad de contacto con maya yucateco (Hernández y Palacios 2015); esto es, los sintagmas nominales definidos, no humanos, inanimados y continuos. Los contextos de mayor accesibilidad referencial son también disparadores del cambio. Los cambios lingüísticos inducidos por contacto son, por tanto, algo más que "interferencias" de la lengua materna (la lengua originaria) en la lengua segunda

18 El sistema local se documenta ya en hablantes bilingües andinos peruanos en el siglo XvI y XVII (Palacios 1996-1997, Palacios 1998b, Palacios 2000a; Rivarola 1995; entre otros). Gómez Seibane (2012) lo documenta en José Santos Vargas, bilingüe boliviano con dominio de español del siglo XIX.

19 No debe olvidarse que estas las variables "factor bilingüismo" y "formas pronominales" están correlacionadas en los estudios de los que he tomado los datos. 
(el español) debidos a un patrón de adquisición incompleto o parcial. Considero, así, que se trata de un cambio indirecto inducido por contacto y no de "interferencias" individuales de cada hablante activadas cada vez que se selecciona una forma pronominal. Esta perspectiva permite dar cuenta de los procesos de creación lingüística de los hablantes de manera sistemática. En esta línea, hago mías las palabras de Martínez (2015: 109) que reclama que la complejidad lingüística y cultural de estas áreas de contacto "reproduce proximidades y alejamientos culturales en distintos espacios comunicativos. En efecto, las soluciones sintácticas que encuentran los hablantes a sus necesidades comunicativas no pueden disociarse de los contenidos con que cada comunidad expresa su lugar en el mundo".

En investigaciones anteriores (Palacios 2005, Palacios 2011, Palacios 2015) he definido los cambios indirectos inducidos por contacto como multicausales, cambios que no suponen importación de material o patrones ajenos a la lengua y en los que la lengua de contacto - las lenguas originarias en nuestro caso actúa como un acelerador de variaciones previamente existentes en la lengua. Así, mediante la influencia indirecta de una lengua en contacto A (la lengua amerindia) con la lengua B (el español) surgen variaciones gramaticales muy significativas que aprovechan la propia evolución interna de esa lengua B para hacer aflorar estrategias gramaticales cuya funcionalidad comunicativa obedece a procesos cognitivos de la lengua A de contacto y que se materializan en las prácticas lingüísticas de la comunidad.

Estos cambios pueden implicar la aceleración de un cambio en proceso y la eliminación de las restricciones lingüísticas que impidan su expansión, la reasignación de nuevos valores a estructuras existentes en la lengua o la reorganización de un sistema completo - como el caso que nos ocupa -, entre otros efectos. Esto se traduce en que los hablantes de variedades distintas pueden hacer categorizaciones diferentes sobre las mismas realidades, lo que tiene su reflejo en la aparición de usos lingüísticos diferenciados. En definitiva, se trata de cambios en los que se perciben reajustes estructurales y funcionales en los que subyacen estructuras cognitivas ajenas al español. El mecanismo que actúa en estos cambios es la convergencia lingüística, entendido como un acercamiento congruente de los rasgos significativos implicados en el cambio, tanto de la lengua amerindia como del español.

La convergencia lingüística reajusta el estatus de las formas pronominales como marcas de concordancia objetiva (lo para objeto directo/le para indirecto) acelerando un proceso lento de la lengua española en el que la concordancia de objeto está sujeta a restricciones considerables (Siewierska 2004). Este proceso implica la neutralización de los rasgos de género en las formas pronominales. Al respecto, es preciso decir que ninguna de las lenguas originarias descritas en §2.1 gramaticaliza el género. Congruentemente con este hecho, las soluciones locales 
emergentes han desechado el género del referente como rasgo condicionante de la selección pronominal de objeto directo. ${ }^{20}$ Esto es, el mecanismo de la convergencia lingüística ha posibilitado en la variedad local la eliminación de distinciones funcionales existentes en el castellano que no son relevantes para las lenguas amerindias. Y esta es una solución sistemática que se repite de manera general en las distintas variedades descritas en §2.1 y que supone un cambio lingüístico que refuerza la distinción de caso, un proceso de gramaticalización en el que las formas pronominales dejan de ser referenciales para consolidarse como concordancias de objeto. Considero, en este sentido, que las variaciones lingüísticas que muestran los hablantes de las zonas de contacto son soluciones novedosas congruentes con las características de su lengua materna que estos hacen emerger en sus repertorios lingüísticos en función de sus necesidades comunicativas.

En cuanto a la neutralización de los rasgos de número, las investigaciones citadas en §2.1 muestran que la pérdida de esta especificación en las formas de objeto directo está presente en todas las variedades de español de contacto descritas, si bien los datos muestran frecuencias menores que las que se aprecian en la de género. Se trata, por tanto, de un proceso situado en una etapa menos avanzada.

Los cambios documentados en las formas locales constatan que estas formas pronominales están en un estadio muy avanzado de gramaticalización hacia su estatus como marcas de concordancia de objeto, lo que las acerca al proceso emprendido por las formas de dativo que se describen para el español en general (le di la noticia a los chicos). Este estatus de las formas pronominales como marcas de concordancia de caso no está muy alejado de lo que ocurre en español, ya que hay una sólida línea de investigación que apunta a que los pronombres átonos de tercera persona tienden a consolidarse en marcas de concordancia objetiva (Bossong 1980; Enrique-Arias 2003; García-Miquel 1991; García Salido 2011; Llorente y Mondéjar 1974; Suñer 1993, entre otros). En otras palabras, se acelera el proceso de gramaticalización presente en el español por el que las formas pronominales pierden su capacidad referencial y evolucionan hacia marcas de concordancia objetiva.

En la sección 2.2 se describen los sistemas leístas de las variedades de contacto con kichwa (español andino ecuatoriano) y guaraní (Paraguay y nordeste argentino). ${ }^{21}$ Estos sistemas tienden hacia una única forma le/s sin especificación de caso, género e incluso de número, lo que indica que se ha completado la reor-

20 Algunas lenguas amazónicas parece que tienen marcas gramaticales de género, sin embargo, no tenemos datos de los sistemas pronominales de hablantes bilingües en estas zonas.

21 En el norte de Perú se documentan casos minoritarios de leísmo con referentes masculinos y animados, similares a los descritos para variedades de español que conservan el sistema pronominal etimológico (Klee y Caravedo 2005; Paredes y Valdez 2008; Valdez Salas 2002; entre otros). 
ganización del paradigma pronominal hacia la reinterpretación funcional de las formas pronominales como concordancias objetivas.

Es interesante constatar que tanto en el español andino ecuatoriano como en Paraguay se han documentado sistemas pronominales en los que la animación funge como disparador del cambio. Son sistemas de transición entre el etimológico y el leísta, como se muestra en el cuadro siguiente.

Cuadro 5: Evolución sistemas pronominales descritos en 2.2.

\begin{tabular}{lll}
\hline & \multicolumn{2}{c}{ Etimológico } \\
\cline { 2 - 3 } & Masc. & Fem \\
\hline OD & $l o / s \quad l a / s$ \\
\hline OI & \multicolumn{2}{|c}{$l e / s$} \\
\hline
\end{tabular}

\begin{tabular}{|c|c|}
\hline \multicolumn{2}{|c|}{ Transición } \\
\hline -Animado & Animado \\
\hline lo/los & le/les \\
\hline \multicolumn{2}{|c|}{ le/les } \\
\hline
\end{tabular}

$\longrightarrow \frac{\frac{\text { Leísta }}{\text { Masc. Fem. }}}{\frac{\text { le/les }}{\text { le/les }}}$

Podríamos aventurar que en estas áreas la primera etapa del cambio fuera un sistema como el que hemos documentado en la sección 2.1, donde la neutralización del género es el disparador del cambio. Sin embargo, no tenemos datos que puedan atestiguarlo, salvo las formas $\mathrm{lo} / \mathrm{s}$ minoritarias documentadas en los hablantes bilingües leístas de Quito y Otavalo, que parecen obedecer a un patrón de animacidad y de neutralización de género (Tablas 13 y 14). Se necesitan estudios diacrónicos para comprobar si realmente ha habido un proceso de gramaticalización como el del Cuadro 5 o si los sistemas de transición y leístas han evolucionado de manera independiente. Lo que parece evidente es que en las formas leístas el disparador del cambio es la animacidad y no tienen cabida las distinciones de género (ni el kichwa ni el guaraní gramaticalizan el género). Por otra parte, es interesante que sean las formas dativas las que terminen imponiéndose como marcas de concordancia objetivas, precisamente las formas que guardan una relación más estrecha con la animacidad.22

Como ya apuntó Thomason (2001), para entender la complejidad de las situaciones de contacto se debe tener en cuenta, entre otros factores, la intensidad y la prolongación del contacto en el tiempo. En el caso del español andino ecuatoriano o el de Paraguay, con situaciones de intensa y continuada convivencia

22 En estas variedades se documenta con profusión la omisión de los objetos directos más prototípicos (accesibles, definidos e inanimados). Palacios (2015) constata en su estudio sobre la omisión en la variedad andina ecuatoriana un 44,8 \% de elisiones en hablantes monolingües de español y un 51,1 \% en bilingües kichwa-español. La omisión del objeto inanimado es la última etapa en el proceso de gramaticalización de las formas pronominales hacia concordancias de objeto. 
histórica de la población indígena y no indígena, los sistemas locales leístas se han extendido a la población no indígena.

En otras zonas de contacto, como hemos referido ya, los hablantes monolingües de español seleccionan también formas pronominales locales en un porcentaje que, en algunos casos, puede ser notable (16,6 \% en zona otomí, 19,2\% en zona maya yucateca, $33,3 \%$ en zona tzutujil, en $56,6 \%$ de zona tsotsil). Evidentemente, se necesita explicar esa variabilidad en las cifras, ya que hay una considerable diferencia entre los porcentajes registrados entre los monolingües de la zona otomí y los de la zona tsotsil, por ejemplo. Esta variación porcentual en las tendencias de uso de los grupos sociales de distintas comunidades dependerá de las características históricas y del perfil sociolingüístico que rodean esas comunidades, lo que significa que hay que apelar también a otros factores explicativos y no únicamente al dominio de la lengua originaria materna, aunque este sea un factor esencial.

Igualmente, es necesario conocer si la lengua está en situación de desplazamiento, como en el caso del otomí o del malecu, y si esto repercute en las actitudes sociales negativas hacia los usos lingüísticos locales. En efecto, la forma lo para objetos directos con referente femenino suele estar indexada con el colectivo indígena y arrastra una evaluación muy negativa. Estos factores pueden ayudar a entender por qué en la zona de San Andrés Cuexcontitlán el grupo monolingüe otomí tiene un $16,5 \%$ de usos locales (Tabla 3) o por qué el palenque Margarita (Tabla 7), el que ha experimentado el mayor desplazamiento de las tres comunidades malecu, es el que muestra porcentajes más bajos de formas locales frente al resto de palenques (24,3\% versus $60 \%$ ). Estos datos contrastan, por el contrario, con los de San Cristóbal de las Casas, donde los usos locales - lo/s - del colectivo monolingüe suponen el 56,6 \% de los casos.

Sin duda en estos procesos, la evaluación social de las formas juega un papel importante. En el español andino ecuatoriano, hay indexación social de la forma local $l o / s$ para referentes femeninos con el colectivo indígena, mientras que la solución le/s se indexa con población urbana no indígena de Quito y se evalúa positivamente. Es relevante, en este sentido, que $l o / s$ sea en la actualidad una forma minoritaria reemplazada por la solución prestigiosa le/s incluso entre colectivos indígenas, como ya señalamos. En estos escenarios complejos, las redes sociales de los hablantes pueden consolidar o no el uso de las formas locales.

El factor escolarización, muy unido a los anteriores, es clave, puesto que la población bilingüe con dominio de la lengua materna es la que muestra menor escolarización, según se constata en la bibliografía. La escuela es un factor determinante para cambiar o imponer patrones de uso; un factor restrictivo y correctivo de la expansión y mantenimiento del cambio que evalúa sistemáticamente 
las formas locales y propende a imponer la norma etimológica. Recordemos, al respecto, cómo Yausaz (2005) constataba una asimetría en el uso del leísmo entre las producciones orales y escritas de los niños correntinos y cómo la relacionaba directamente con la evaluación negativa que los docentes de Corrientes hacían sobre este fenómeno.

En definitiva, todos estos factores, densamente entrelazados, son los que pueden explicar de manera coral la compleja situación lingüística de cada variedad. Se necesita, por tanto, repensar la idea de que los hablantes usan las formas locales únicamente en función de su mayor o menor destreza en la lengua originaria y el español. Resulta más congruente con los datos pensar que en la misma zona coexisten distintos sistemas y que esos patrones pronominales distintos están disponibles para los hablantes, que pueden seleccionar los elementos de ambos repertorios lingüísticos en función de factores como los expuestos en estas páginas, pero también de sus necesidades comunicativas.

\section{A modo de conclusiones: algunas implicaciones teóricas y metodológicas}

Partimos de una perspectiva teórica que concibe las gramáticas de las lenguas como sistemas dinámicos donde los hablantes categorizan modos de representar la realidad; en las zonas de contacto lingüístico, la coexistencia de lenguas puede conllevar distintos modos o sistemas de categorización que podrían manifestarse en variaciones lingüísticas significativas en las variedades de lengua que usan los hablantes de esas zonas bilingües. Consideramos, además, que las distintas variedades de una lengua también tienen gramáticas que difieren entre sí. En el caso que nos ocupa, hemos visto que en una misma variedad de español conviven patrones pronominales diferentes y que hay grupos sociales que comparten la misma gramática, pero no otros, o no en la misma proporción, lo que exige analizar la variación intra-hablante para poder reconstruir su sistema pronominal. Esto supone girar la perspectiva de la investigación, que ya no se centrará únicamente en los efectos del contacto, sino en el hablante inmerso en su ecología y en sus producciones de habla real.

Esto conlleva implicaciones metodológicas que no por evidentes dejan de ser importantes: 1) la necesidad de realizar trabajos de campo a partir de datos de habla real extraídos en su contexto natural; 2) que estos trabajos de campo se realicen no solo en diferentes variedades de español en contacto, sino también en comunidades distintas de una misma área de contacto (los datos confirman que la evolución de los sistemas pronominales no es igual en el área andina ecuatoriana 
y peruana, por ejemplo); 3) se necesitan protocolos similares y condiciones de comparabilidad para que los datos sean equiparables y se puedan extraer conclusiones generalizables; 4) es preciso entender la complejidad de las comunidades de habla como un continuo complejo donde se superponen hablantes con distinto grado de bilingüismo, lo que implica la coexistencia de varias modalidades de habla como práctica cotidiana; un continuo de uso de carácter gradual, no discreto, de estas situaciones de contacto de lenguas y su complejidad como el que se muestra en el Gráfico 1.

Gráfico 1: Continuo de modalidades lingüísticas en situaciones de contacto.

Monolingües lengua indígena $\leftrightarrow$ bilingües dominante lengua indígena $\leftrightarrow$ bilingües simétricos $\leftrightarrow$ bilingües dominantes $\leftrightarrow$ monolingües español

En cuanto a implicaciones teóricas para perfilar mejor la teoría del contacto, podemos apuntar las ideas que esbozamos a continuación, resultado de los datos analizados.

1. Las gramáticas de lenguas tipológicamente muy distantes pueden ser modeladas con soluciones novedosas que se erigen en recursos altamente productivos congruentes con las lenguas implicadas. En efecto, en el contacto de lenguas lo relevante son las similitudes estructurales y las equivalencias funcionales que los hablantes perciben entre las lenguas implicadas, y son estas las que determinan la dirección de los cambios indirectos inducidos por contacto. Para ello, parto del supuesto de que el hablante bilingüe percibe similitudes y diferencias entre las lenguas implicadas (reales o imaginadas), como apuntan Jarvis y Pavlenko (2008), y que esa percepción hace emerger soluciones novedosas que pueden consolidarse en cambios lingüísticos inducidos por contacto. Así, considero que el hablante acerca su variedad de castellano a la lengua indígena y aprovecha las potencialidades de los sistemas para explotar nuevas estrategias comunicativas mediante el mecanismo de la convergencia lingüística. En el caso que nos ocupa, el hablante bilingüe percibe similitudes entre el castellano y su lengua materna, y hace converger las formas pronominales con sus intereses y estrategias. La ausencia de gramaticalización de género en las lenguas amerindias y en las formas pronominales de objeto indirecto orienta la evolución de los sistemas pronominales de estas zonas de contacto.

2. Los cambios indirectos inducidos por contacto que tienen lugar en una lengua explotan las potencialidades del sistema de esa lengua. Volviendo a los sistemas pronominales, la reorientación de las formas pronominales hacia marcas de con- 
cordancia objetiva supone una ampliación de las posibilidades que ya ofrece el castellano. Las lenguas indígenas actúan como acelerador del proceso de gramaticalización de las formas pronominales como marcas de concordancia objetiva que ya está presente en español.

3. El cambio inducido por contacto tiene una etapa de creación y otra de difusión. Se inicia en el grupo de los bilingües con lengua amerindia dominante, y se va expandiendo entre los bilingües simétricos, los bilingües con español dominante y llega hasta los monolingües. Esta etapa de difusión del cambio es progresiva y está favorecida por el factor "lengua dominante".

4. La comparabilidad de un mismo fenómeno desde una perspectiva pandialectal permite contemplar la evolución gradual de un cambio lingüístico de manera sincrónica. En el caso de los sistemas pronominales, esta perspectiva reproduce las distintas etapas del proceso de gramaticalización inducida por contacto, desde los sistemas que aún no han registrado cambios lingüísticos (sistema etimológico) hasta los sistemas más evolucionados (sistema leísta), pasando por sistemas que permanecen en un estado de evolución intermedio (sistema de caso sin especificación de género/número y sistema de transición).

5. Cuestiono la premisa de que los cambios inducidos por contacto producen resultados simplificados. Hablamos insistentemente de simplificación de los paradigmas pronominales y esa terminología conlleva la idea de reducción de formas y de significados, y suele estar vinculada con estrategias de adquisición incompleta. Creo que es preciso plantearse si la simplificación morfológica de las formas no implica procesamientos más complejos para que los interlocutores asignen las referencias adecuadas y la comunicación sea satisfactoria y eficaz. La simplificación morfológica implica, paradójicamente, una complejidad cognitiva relacionada con la gramaticalización de formas de concordancias de objeto. $\mathrm{Si}$ las formas pronominales dejan de ser referenciales, ¿cómo se asigna la referencia con el antecedente? Es posible que, en este sentido, los hablantes utilicen las soluciones emergentes como estrategias de máxima eficiencia que permitan nuevos significados pragmáticos discursivos. La finalidad de estos cambios inducidos por contacto suele obedecer a estrategias comunicativas que permiten una mejor explotación de los recursos lingüísticos que el hablante bilingüe tiene a su alcance.

6. La evaluación social de los fenómenos lingüísticos y la escuela como institución son factores reguladores de la expansión, el mantenimiento o la desaparición de estos cambios y deben considerarse a la hora de explicarlos. 
Por otra parte, necesitamos nuevas investigaciones que corroboren si las soluciones emergentes de los cambios inducidos por contacto siguen siempre parámetros, jerarquías o principios universales. Así ocurre en la neutralización del rasgo de género de las formas pronominales locales descritas en §2.1. Esto implica que las formas de dativo orientan el cambio, dado que las de acusativo "copian" las de dativo, inespecificadas para el género. Sin embargo, en los sistemas pronominales referenciales del centro y norte de España, las formas de acusativo orientan el cambio y el resultado es un sistema con un patrón de género tanto para las formas acusativas como para las dativas (FernándezOrdóñez 2001). Al respecto, Elvira (1998: 227) propone que "el cambio analógico está orientado desde las formas menos marcadas o más frecuentes hacia las marcadas o menos frecuentes"; esto es, el caso que prevalece en los procesos de cambio, según las tendencias universales de cambio, es precisamente el no marcado, ya que los procesos de cambio analógico parece que están orientados a favor de las formas no marcadas. Si entendemos que las formas menos marcadas son las de acusativo - el dativo sería el caso marcado ya que es menos nuclear que el acusativo, distribucionalmente es menos restringido y tiene menos diferencias formales -, el cambio inducido por contacto que hemos analizado en estas páginas avanza en una dirección opuesta al ocurrido en los sistemas referenciales de España.

Igualmente, es preciso comprobar si en otras variedades de contacto la expansión del cambio avanza entre los distintos grupos sociales a partir de las categorías más prototípicas, como mostraban Hernández y Palacios (2015) en el caso del español en contacto con maya yucateco, donde el cambio se inicia en los objetos directos prototípicos, inanimados y continuos; cuando esta etapa se consolida con una alta frecuencia y resulta no marcado, se extiende a otros contextos en etapas sucesivas, primero a las entidades discontinuas y, posteriormente, a las entidades inanimadas.

En definitiva, se necesitan más investigaciones basadas en datos de habla natural para que podamos explicar la complejidad de las situaciones de contacto lingüístico.

\section{Referencias bibliográficas}

Aguado Orea, Javier. 2000, Adquisición de los complementos pronominales personales en español. Madrid: Universidad Autónoma de Madrid.

Avelino, Rosnátaly. 2017. Contacto lingüístico entre el español y el otomí en San Andrés Cuexcontitlán. Ciudad de México: Tesis de licenciatura de la UNAM. 
Avelino, Rosnátaly. 2021. La neutralización de género y número en los clíticos de tercera persona de acusativo en el español en contacto con otomí. En Élodie Blestel y Azucena Palacios (eds.), Variedades del español en contacto con otras lenguas. Berlín: Peter Lang. 76-95.

Bossong, Georg. 1980. Aktantenfunktionen in romanischen Verbalsystem. Zeitschrift für romanische Philologie 96. 1-22.

Calvo Pérez, Julio. 1996-97. Pronominalización en español andino. Ley de mínimos e influencia del quechua y del aimara. Homenaje al Dr. Germán de Granda. Anuario de Lingüística Hispánica XII-XII(II). 521-544.

De Granda, Germán. 1982. Origen y formación del leísmo en el español del Paraguay. Ensayo de un método. Revista de Filología Española 52. 259-283.

Domínguez, Laura. 2006. La adquisición de pronombres átonos en español. En Marian Amengual, María Juan y Joana Salazar (eds.), Adquisición y enseñanza de lenguas en contextos plurilingües. Ensayos y propuestas aplicadas, 67-76. Palma: Universitat de les Illes Balears.

Elvira, Javier. 1998. El cambio analógico. Madrid: Gredos.

Enrique-Arias, Andrés. 2003. From clitics to inflections: diachronic and typological evidence for affixal object agreement marking in Spanish. En Bernard Fradin (ed.), Forum de Morphologie (3e. rencontres), 67-75. Lille: Université de Lille.

Fernández-Ordóñez, Inés. 2001. Hacia una dialectología histórica. Reflexiones sobre la historia del leísmo, el laísmo y el loísmo. Boletín de la RAE LXXXI, 389-464.

Franco, Jon. 2000. Agreement as a Continuum: The case of Spanish pronominal clitics. En Frits

Beukema y Den Dikken (eds.), Clitic Phenomena in European Languages, 147-190. Amsterdam: John Benjamins.

García-Miguel, José M. 1991. La duplicación de complementos directos e indirectos como concordancia. Verba 18. 375-410.

García Salido, Marcos. 2011. Pronombres y afijos personales. Estudio con datos de español Conversacional. Santiago de Compostela: Tesis doctoral de la Universidad de Santiago de Compostela.

García Tesoro, Ana Isabel. 2010. Español en contacto con el tzutujil en Guatemala: cambios en el sistema pronominal átono de tercera persona. Revista Internacional de Lingüística Iberoamericana VIII(2). 133-155.

García Tesoro, Ana Isabel. 2018. El sistema pronominal átono de tercera persona en la variedad de contacto con el tzutujil: hacia una concordancia de objeto. Revista Internacional de Lingüística Iberoamericana XVI(2). 83-96.

García Tesoro, Ana Isabel y Víctor Fernández-Mallat. 2015. Cero vs. Lo en español andino (Chinchero, Cuzco). Círculo de lingüística aplicada a la comunicación 61. 131-157.

Godenzzi, Juan C. 1986. Pronombres de objeto directo e indirecto del castellano en Puno. Lexis X. 187-202.

Gómez Seibane, Sara. 2012. Neutralización de género, omisión y duplicación de objetos en el español boliviano de la independencia (1814-1825). En José L. Ramírez Luengo (ed.), Por sendas ignoradas. Estudios sobre el español del siglo xIx, 75-97. Lugo: Axac.

Guillán, M. Isabel. 2012. Procesos de cambio lingüístico inducidos por contacto en el español del nordeste de Argentina: el sistema pronominal átono. Madrid: Tesis Doctoral de la UAM.

Haboud, Marleen y Esmeralda de Vega. 2008. Ecuador. En Azucena Palacios (ed.), El español en América. Contactos lingüísticos en Hispanoamérica, 161-188. Barcelona: Ariel. 
Hernández, Edith. 2017. Los pronombres de objeto indirecto en el español de contacto con el maya yucateco y el fenómeno de la discordancia. En Azucena Palacios (ed.), Variación y cambio lingüístico en situaciones de contacto, 161-184. Fráncfort y Madrid: Vervuert/ Iberoamericana.

Hernández, Edith y Azucena Palacios. 2015. El sistema pronominal átono en la variedad de español en contacto con maya yucateco. Círculo de lingüística aplicada a la comunicación 61. 36-78.

Hernández Pina, Fuensanta. 1990. Teorías psico-sociolingüísticas y su aplicación a la adquisición del español como lengua materna. Madrid: S. XXI.

Jarrín Elena G. 2019. El sistema pronominal átono de tercera persona de un grupo de hablantes de la parroquia Calderón de la ciudad de Quito. TFM. Madrid: UAM.

Jarvis, Scott y Aneta Pavlenko. 2008. Crosslinguistic Influence in Language and Cognition. Nueva York y Londres: Routledge.

Klee, Carol A. 1989. The acquisition of clitic pronouns in the Spanish interlanguage of Quechua speakers: A contrastive case study. Hispania 72. 402-408.

Klee, Carol A. 1990. Spanish-Quechua language contact: The clitic pronoun system in Andean Spanish. Word 41. 35-46.

Klee, Carol A. y Rocío Caravedo. 2005. Contact-Induced language change in Lima, Peru: The case of clitic pronouns. En David Eddington (ed.), Selected Proceedings of the 7th Hispanic Linguistics Symposium, 12-21. Somerville, MA: Cascadilla Proceedings Project.

Lafford, Barbara y Joseph Collentine. 1987. Lexical and grammatical access errors in the speech of intermediate/advanced level students of Spanish. Lenguas Modernas 14. 87-112.

López Gutiérrez, Eva M. 2018. Sistema pronominal átono de objeto directo en el español de monolingües (español) y bilingües (tsotsil-español) de San Cristóbal de las Casas, Chiapas. México: Tesis de licenciatura de la Escuela Nacional de Antropología e Historia.

Llorente, Antonio y José Mondéjar. 1974. La conjugación objetiva en español. Revista Española de Lingüística 4(1). 1-60.

Martínez, Angelita. 2000. Estrategias etnopragmáticas en el uso de los pronombres clíticos lo, la y le, en la Argentina, en zonas de contacto con lenguas aborígenes. Leiden: University of Leiden Press.

Martínez, Angelita. 2013. Los pronombres clíticos lo, la, le en el español de la Argentina. En Laura Collantoni y Celeste Rodríguez Louro (eds.), Perspectivas Teóricas y Experimentales sobre el español argentino, 397-416. Madrid: Iberoamericana.

Martínez, Angelita. 2015a. ¿Cómo afecta la cultura a la gramática? El caso de los clíticos en el español americano. Círculo de Lingüística Aplicada a la Comunicación 61. 186-210.

Martínez, Angelita. 2015b. Las escuelas del Mercosur: la trama de las gramáticas y el concepto de identidades dinámicas. En Elvira Arnoux y Roberto Bein (eds), Política lingüística y enseñanza de lenguas, 109-134. Buenos Aires: Ed. Biblos.

Palacios, Azucena. 1996-97. Un caso de bilingüismo histórico: aspectos lingüísticos de la obra de Santacruz Pachacuti. Homenaje al Dr. Germán de Granda. Anuario de Lingüística Hispánica XII-XIII(1). 397-412.

Palacios, Azucena. 1998a. Variación sintáctica en el sistema pronominal del español paraguayo. Anuario de Lingüística Hispánica XIV. 451-474.

Palacios, Azucena. 1998b. A propósito de la llamada falsa pronominalización en español andino: la crónica de Santacruz Pachacuti. Lexis XXII(2). 119-146. 
Palacios, Azucena. 2000a. Apuntes sobre la historia del español americano: La lengua de un cronista indio del siglo XVII. Analecta Malacitana 23(2). 639-656.

Palacios, Azucena. 2000b.El sistema pronominal del español paraguayo: un caso de contacto de lenguas. En Julio Calvo Pérez (ed), Teoría y práctica del contacto: el español de América en el candelero, 123-144. Fráncfort y Madrid: Vervuert/Iberoamericana.

Palacios, Azucena. 2005. Aspectos teóricos y metodológicos del contacto de lenguas: el sistema pronominal del español en áreas de contacto con lenguas amerindias. En Volker Noll, Klaus Zimmermann e Ingrid Neumann-Holzschuh (eds.), El español en América: Aspectos teóricos, particularidades, contactos, 63-94. Fráncfort: Vervuert.

Palacios, Azucena. 2006. Cambios inducidos por contacto en el español de la sierra ecuatoriana: la simplificación de los sistemas pronominales (proceso de neutralización y elisión). Tópicos de seminario. Huellas del Contacto Lingüístico 15. 197-230.

Palacios, Azucena. 2008. Paraguay. En Azucena Palacios (ed.), El español en América: contactos lingüísticos en Hispanoamérica, 279-300. Barcelona: Ariel.

Palacios, Azucena. 2011. Nuevas perspectivas en el estudio del cambio inducido por contacto: hacia un modelo dinámico del contacto de lenguas. Lenguas Modernas 38. 17-36.

Palacios, Azucena (ed.). 2015. El sistema pronominal átono de 3a persona: variedades de español en contacto con otras lenguas. Círculo de Lingüística Aplicada a la Comunicación 61. Número monográfico.

Paredes, Liliana y María Luz Valdez. 2008. Language contact and change: Direct object leísmo in Andean-Spanish. En Maurice Westmoreland and Juan Antonio Thomas (eds.), Selected Proceedings of the 4th Workshop on Spanish Sociolinguistics, 140-148. Somerville, MA: Cascadilla Proceedings Project.

Pueyo, Francisco. 1992. El sistema de clíticos en niños bilingües de Los Angeles: transferencia lingüística y motivación social. En Hernán Urrutia Cárdenas y Carmen Silva-Corvalán (eds), Bilingüismo y adquisición del español. Estudios en España y EE. UU., 255-274. Bilbao: Instituto Horizonte.

Rivarola, José L. 1995. Aproximación histórica a los contactos de lenguas en el Perú. En Klaus Zimmermann (ed.), Lenguas en contacto en Hispanoamérica: nuevos enfoques, 135-159. Madrid: Iberoamericana.

Sánchez Avendaño, Carlos. 2015. El sistema pronominal átono de 3a persona en el español hablado por los malecus de Costa Rica. Círculo de Lingüística Aplicada a la Comunicación 6. 79-103.

Sánchez Paraíso, María. 2017. El sistema pronominal átono de tercera persona en el español andino de la zona rural de Juliaca (Perú). Ponencia presentada en el XVIII Congreso Internacional de ALFAL. Bogotá.

Siemund, Peter y Noemi Kintana (eds.). 2008. Language Contact and Contact Languages. Amsterdam y Filadelfia: John Benjamins Publishing Company.

Siewierska, Anna. 2004. Person. Cambridge: Cambridge University Press.

Suñer, Margarita. 1993. El papel de la concordancia en las construcciones de reduplicación de clíticos. En Olga Fdez. Soriano (ed.). Los pronombres átonos, 174-204. Madrid: Taurus.

Symeonidis, Haralambos. 2013. Análisis sociolingüístico del leísmo en el español paraguayo. Revista Internacional d'Humanitats 27(jan-abr). 55-68.

Thomason, Sarah G. 2001. Language Contact. Edinburg: University Press.

Torres Sánchez, Nadiezdha. 2015. El sistema pronominal en el español de bilingües tepehuano del sureste-español. Círculo de Lingüística Aplicada a la Comunicación 61. 10-35. 
Torres Sánchez, Nadiezdha. 2018. “Aquí hablamos tepehuano y allá español”. Un estudio de la situación de bilingüismo incipiente entre español y tepehuano del sureste en Santa María Ocotán y Durango. Ciudad de México: Tesis doctoral del Colegio de México.

Valdez Salas, María Luz. 2002. Clitics in the Speech of Monolingual Andean Spanish Speakers. Pittsburgh, PA: Tesis doctoral de la University of Pittsburgh.

Yausaz, Fabián. 2005. Estrategias de adecuación en el uso de los pronombres clíticos en textos orales y escritos producidos por hablantes correntinos. Revista Estudios en Ciencias Humanas 7. 1-12. 